\title{
Work orders management based on XML file in printing
}

\author{
Peipei $\operatorname{Ran}^{1},{ }^{*}$, Wenjie Yang ${ }^{1}$, Zhongyue $\mathrm{Da}^{1}$, and Yuke Huo ${ }^{1}$ \\ ${ }^{1}$ School of Printing and Packing Engineering, Beijing Institute of Graphic Communication, Beijing, \\ China
}

\begin{abstract}
The Extensible Markup Language (XML) technology is increasingly used in various field, if it's used to express the information of work orders will improve efficiency for management and production. According to the features, we introduce the technology of management for work orders and get a XML file through the Document Object Model (DOM) technology in the paper. When we need the information to conduct production, parsing the XML file and save the information in database, this is beneficial to the preserve and modify for information.
\end{abstract}

\section{Introduction}

Extensible Markup Language, short for XML, announced in 1998 by the W3C, become a universal format for EDI (Electronic Data Interchange) and replace the Standard Generalized Markup Language (SGML) and Hyper Text Mark-up Language (HTML) due to the advantages such as easily used, strong expansibility, data and expression separated, simple data exchange. XML is a versatile and well-operational data exchange technology that enables heterogeneous compatibility with information systems and ensures efficient transfer, exchange, sharing and integration of data between data modules [1]. The role of XML file is: the first is application' configuration file, the second is used to exchange data between different language, the third is as a database to save data [2]. The work orders management is core module of Enterprise Resource Planning (ERP) in printing industry. In actual production, we can import the XML file that from other enterprises into the local ERP system to conduct production, or send the work order information to other enterprises in the form of XML files.

\section{Management of work orders}

The system of work orders management is development on the foundation of technology such as Java, DOM, XML and database, the framework for development technology is shown as figure 1 .

1 Corresponding author: 376007414@qq.com 


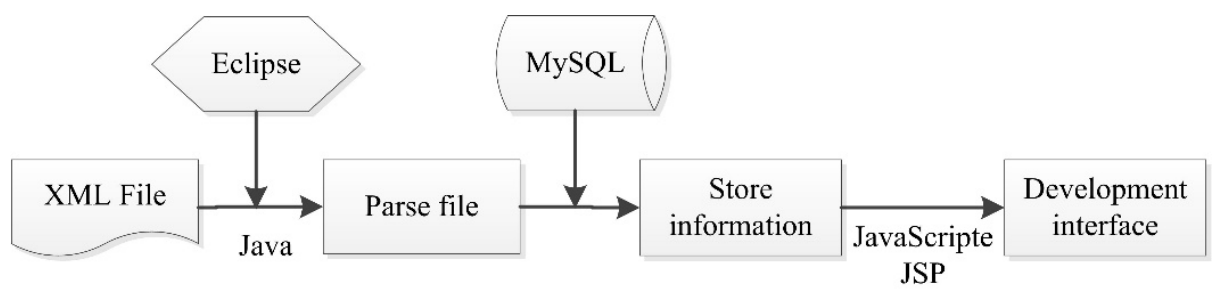

Fig.1. The framework for development technology

First, the client can provide a XML file which contains the product structure, printing effect and contact information to printing industry. Second, the industry receive the XML file and start to parse the file and save the basic information for jobs and description information for process in database. Third, the system store the work orders information through MySQL database, including the basic information of work orders, parts, process and description.

\section{The parsing technology}

\subsection{The execution rote for $\mathrm{XML}$ file parsing}

The XML file is a kind of tree structure consisting of different type nodes. The top nodes is usually used to describe the purpose and requirements that the entire production achieves, also said product information description. The center nodes, also said process group nodes, refer to the process of center production, for example, the printing of hardcover book includes the process of platemaking, printing, book-cover making and post-press finishing, each process can be regarded as an center node. Each center node contains nodes, also said process nodes, which represent a specific, single, simple process that required to complete an intermediate product [3].

There are two methods for parsing of XML file: the first is to read or modify the information according to the DOM tree structure that composed of relationship between different type nodes. The second is to read the information of XML file in order, also read the needed data. The paper mainly introduces how to create, modify, query the XML file through the first method (as shown in figure 2), beginning with the root node to parse different types nodes that constitute the entire production process, including the nodes describing the product information and process.

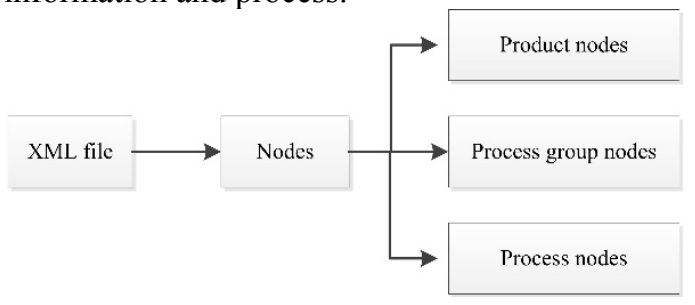

Fig.2. The execution rote for XML file parsing

\subsection{The technology of DOM}

Document Object Model, short for DOM, is a standard independent to language and platform and developed by $\mathrm{W} 3 \mathrm{C}$, this standard provides an interface that can be used in a variety of programming languages, operating systems and platforms, it also defines the logical structures of document and the methods to preserve and modify document [4]. 
DOM technology can transfer XML file into a document object model of tree structure, the user can dynamically create document and traverse the structure of document through interviewing the object model [5]. The data in document can be modified, deleted and added. Its working principle is shown in figure 3.

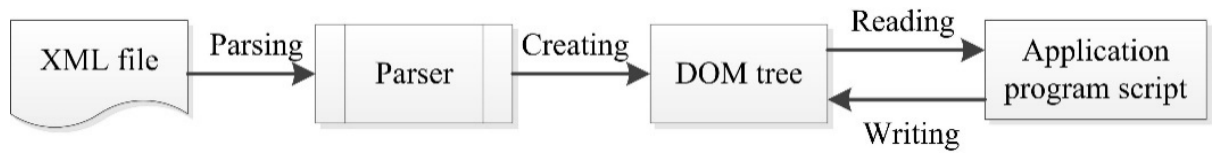

Fig.3. The working principle of DOM

\section{The creating and parsing for XML file}

First, in order to expediently share information between different ERP systems, based on security of information transfer, using DOM technology to create XML file according to information of work orders, as shown in Figure 2.

The XML file for work orders:

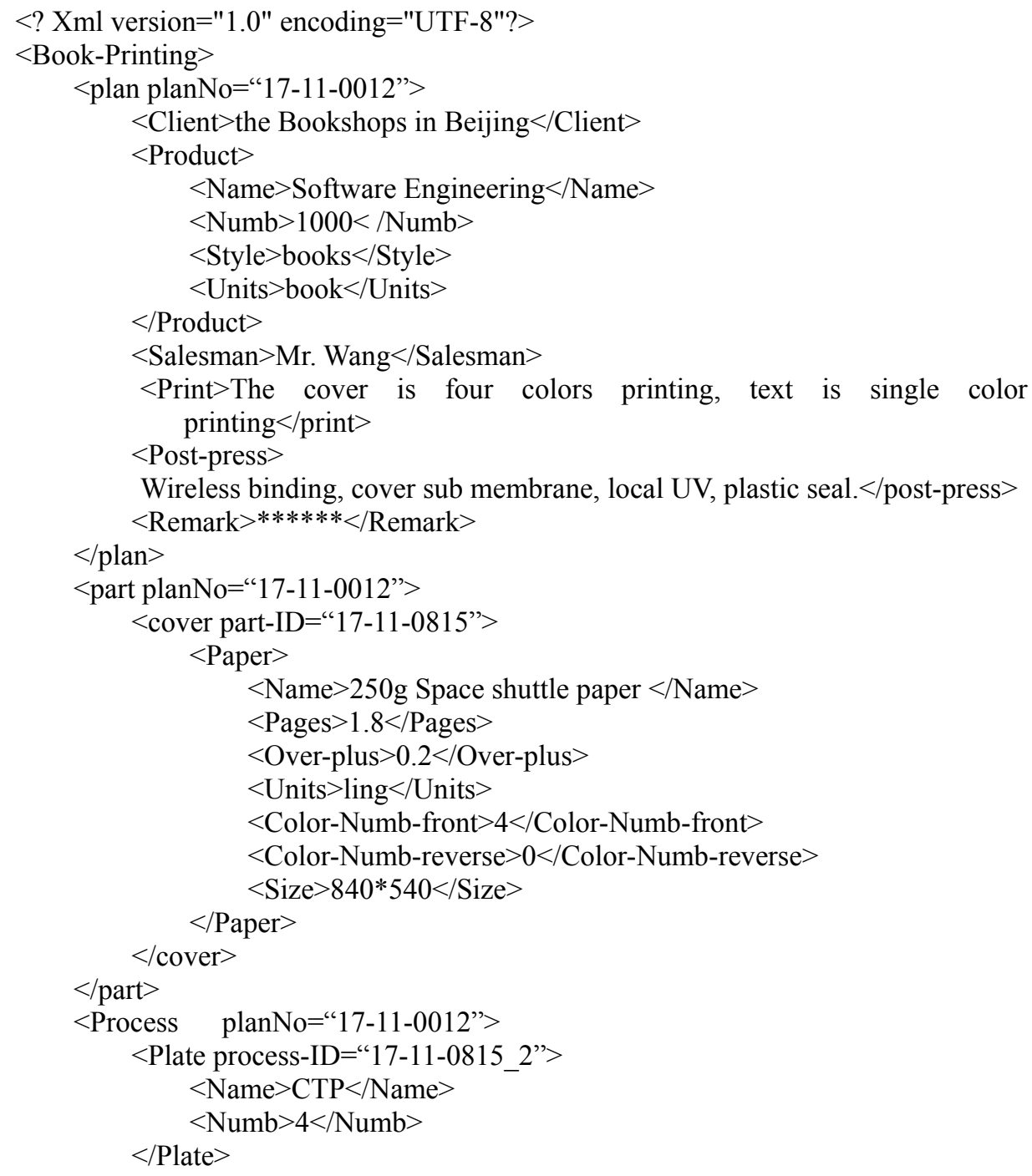




\section{$<$ Process_1 process-ID =“17-11-0815_21"> \\ Single surface optical film $<$ Process_1 $>$ \\ $<$ Process_2 process-ID $=$ “17-11-0815_22> \\ Bronzing $<$ Process_2 $>$ $<$ Process $>$}

$</$ Book-Printing $>$

This file contains four parts: basic information of work orders, part information, process information and description of printing information, these parts are linked by ID. The "plan" node and its child nodes include basic information of work orders, for example the information of client and product. The "part" node and its child nodes refer to the basic part information for production, such as the name, specification, number of paper in the printing of cover. The "process" node and its child nodes consist refer to the process information consisting of process of part production, such as plate and the process in post-press.

Second, the data in the file is obtained through the parsing technology, the process is shown in figure 4.

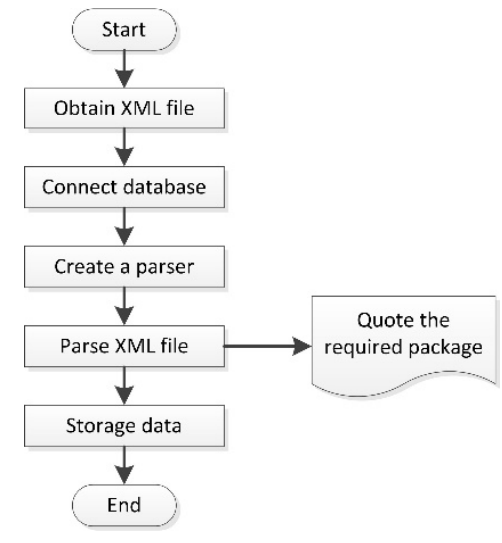

Fig.4. The process of parsing XML file

Third, the data obtained from the XML file is stored in database, it can be browsed by user in the interface of work orders management system, as shown in figure5.

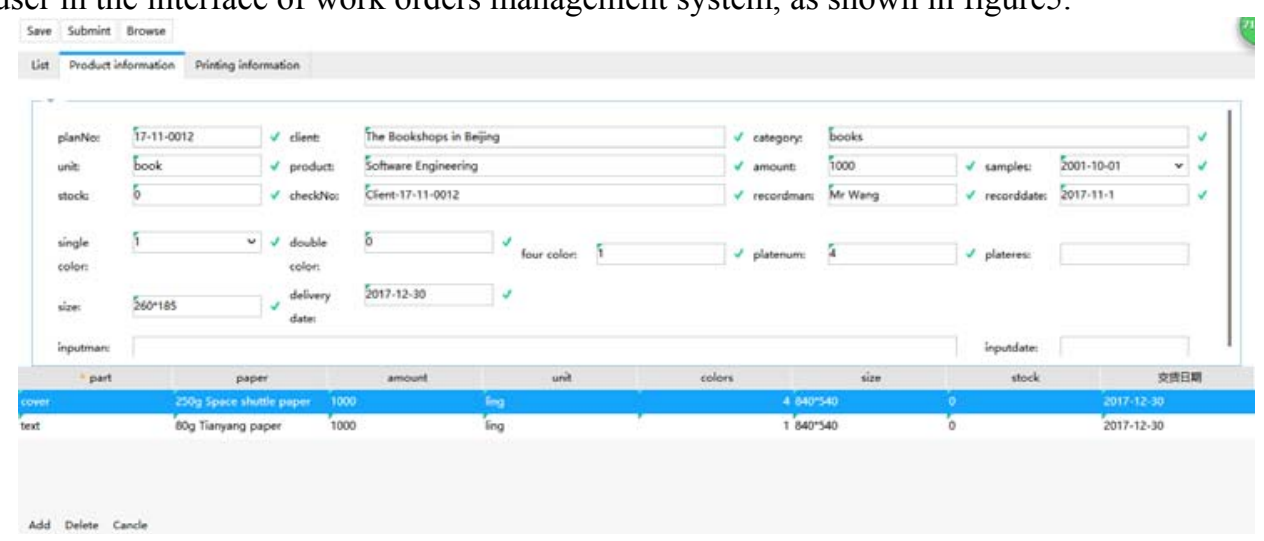

Fig.5. The interface of work orders management

The figure 5 display the interface of work orders management, including the detail information for client, product and printing process. Users can directly add or modify information on this interface, or make changes through the XML file. 


\section{Conclusion}

Printing production is composed of a series of independent processes, which rely on a variety of data to contact for each other. In different stages of production, various types of information in order to pass from receiving the order to production to logistics and distribution, the production process become more complicated due to exchange of information, which directly leads to the production of low efficiency and high consumption. The work orders management, as core module of ERP in printing industry, in order to facilitate the exchange of information between the production departments or enterprises, using DOM technology to transfer woke orders information into XML files, the XML file can be encrypted, compared with the traditional word file, the information is safer and hard to leak. It can be transmitted between different systems in companies, the production sectors or enterprises only need to resolve the information they need to carry out production guidance, this can reduce the waste of the time, but also can improve the efficiency of production and management.

Acknowledgment: The study is supported by the National Key Scientific Instrument and Equipment Development Project (No.2013YQ140517).

\section{Reference}

1. Feifei Chen. The Research and Application of XML Document Resolution Technology Based on DOM4J [J]. Software Guide, 2016, 15(3).

2. Yi Sun. The development and research of XML integrated editing and analysis environment [D]. Wuhan: Wuhan University of Technology, 2003.

3. Jie Shi. The design of printing industry orders management system for supporting JDF [D]. Beijing: Beijing Institute of graphic communication, 2011.

4. Shuyi Fan, Yan Li, Chen Meng. Application of SAX and DOM in XML file parsing [J]. Microcomputer Applications, 2011, 12 (27).

5. JingYang, ShuangeZhou. A conversion method forunstructured data based on XML [J]. Computer Science, 2017, 11 (44). 